International Mathematical Forum, 2, 2007, no. 39, 1917 - 1919

\title{
Injectivity of the Determinantal Map for Space of Sections of Stable Vector Bundles on Curves
}

\author{
E. Ballico ${ }^{1}$ \\ Dept. of Mathematics \\ University of Trento \\ 38050 Povo (TN), Italy \\ ballico@science.unitn.it
}

\begin{abstract}
Let $X$ be a smooth aprojective curve of genus $g \geq 2$ and $E$ a rank $n$ vector bundle on $X$. For any linear subspace $W$ of $E$ let $\tau_{E, W}$ : $\bigwedge^{n}(W) \rightarrow H^{0}(X, \operatorname{det}(E))$ denote the exterior product map. Here we study the existence of triple $(X, E, V)$ such that $E$ is stable and $\tau_{E, W}$ is injective, essentially when $g \gg n$.
\end{abstract}

Mathematics Subject Classification: $14 \mathrm{H} 60$

Keywords: stable vector bundle,; vector bundles on curves; Grassmannian; Plücker embedding

Let $X$ be a smooth and connected projective curve of genus $g \geq 2$ and $E$ a rank $n$ vector bundle on $X$. For any linear subspace $W$ of $E$ let $\tau_{E, W}$ : $\bigwedge^{n}(W) \rightarrow H^{0}(X, \operatorname{det}(E))$ denote the exterior product map. The study of injectivity of $\tau_{E, W}$ was started by N. Teixidor i Bigas in [4]. She also linked it the Plüker embedding of the Grassmannian. Here (stimulated by [3]. §4) we will consider it again and prove the following result.

Theorem 1. Fix integers $n, b, k, g, d$ such that $b>n \geq 2, g \geq 2 k-1 \geq 3$, set $u:=\left\lceil\left(\begin{array}{l}b \\ n\end{array}\right) / n\right\rceil$. Assume $d \geq g+u k n+1$. Let $X$ be a smooth and connected $k$-gonal curve of genus $g$. Fix $L \in P i c^{d}(X)$. Then there exist a rank $n$ stable vector bundle $E$ and a b-dimensional linear subspace $V$ of $H^{0}(X, E)$ such that $\operatorname{det}(E) \cong L$ and the exterior product map $\tau_{E, V}: \bigwedge^{n}(V) \rightarrow H^{0}(X$, $\operatorname{det}(E))$ is injective.

Theorem 2. Fix integers $n, b, k, g, d$ such that $b>n \geq 2, g \geq 2 k-1 \geq 3$, set $u:=\left\lceil\left(\begin{array}{l}b \\ n\end{array}\right) / n\right\rceil$. Assume $d \geq u k n+n+1$. Let $X$ be a smooth and connected

\footnotetext{
${ }^{1}$ The author was partially supported by MIUR and GNSAGA of INdAM (Italy).
} 
$k$-gonal curve of genus $g$. There is an integral family $U \subseteq \operatorname{Pic}^{d}(X)$ such that $\operatorname{dim}(A)=\min \{g, d-u k n\}$ and for all $L \in A$ the following is true. Then there exist a rank $n$ stable vector bundle $E$ and a b-dimensional linear subspace $V$ of $H^{0}(X, E)$ such that $\operatorname{det}(E) \cong L$ and the exterior product map $\tau_{E, V}: \bigwedge^{n}(V) \rightarrow$ $H^{0}(X, \operatorname{det}(E))$ is injective.

Remark 1. The case $b=2 n+1, d=2 g-2$ of Theorem 1 give a partial extension ( $E$ is not spanned in Theorem 1 ) to the case $n \geq 3$ of [3], part (ii) of Theorem 4.1. However, here we also get the existence part for stable vector bundles, while in [3] only semistable and decomposable solutions were given. We are also more explicit on the choice of the curve $X$.

Remark 2. Here we will follow [2], §2. For all integers $b>a>0$ let $G(a, b)$ denote the Grassmannian of all $(b-a)$-dimensional linear subspaces of $\mathbb{K}^{\oplus b}$. Thus $\operatorname{dim}(G(a, b))=a(b-a), \operatorname{Pic}(G(a, b)) \cong \mathbb{Z}$ and the positive generator $\mathcal{O}_{G(a, b)}(1)$ of $\operatorname{Pic}(G(a, b))$ induces the Plücker embedding $j_{a, b}$ of $G(a, b)$ into $\mathbf{P}^{N(a, b)}, N(a, b):=\left(\begin{array}{l}b \\ a\end{array}\right)-1$. To simplify the notations we will identify $G(a, b)$ with $j_{a, b}(G(a, b))$. Let

$$
0 \rightarrow S_{a, b} \rightarrow \mathcal{O}_{G(a, b)}^{\oplus n} \rightarrow Q_{a, b} \rightarrow 0
$$

be the tautological exact sequence of $G(a, b)$. Thus $Q_{a, b}$ is the rank $a$ tautological quotient bundle of $G(a, b), S_{a, b}$ is the tautological rank $(b-a)$ subbundle of $G(a, b), \operatorname{det}\left(Q_{a, b}\right) \cong \operatorname{det}\left(S_{a, b}\right)^{*} \cong \mathcal{O}_{G(a, b)}(1)$ and $T G(a, b) \cong Q_{a, b} \otimes S_{a, b}^{*}$. Set $V_{a, b}:=H^{0}\left(G(a, b), Q_{a, b}\right)$. Hence $\operatorname{dim}\left(V_{a, b}\right)=b$ and the determinantal map $\tau_{a, b}: \bigwedge^{a}\left(V_{a, b}\right) \rightarrow H^{0}\left(G_{a, b}, \mathcal{O}_{G(a, b)}(1)\right)$ is bijective. For every closed subscheme $T \subset G(a, b)$ let $V_{T, a, b}$ denote the image og the restriction map $H^{0}\left(G(a, b), Q_{a, b}\right) \rightarrow H^{0}\left(T, Q_{a, b} \mid T\right)$. A curve $X$ in a projective space $\mathbf{P}^{N}$ is called a chain of $d$ lines if it is reduced, connected, nodal, it has exactly $d$ irreducible components $C_{1}, \ldots, C_{d}$, each of them being a line, and $C_{i} \cap C_{j} \neq \emptyset$ if and only if $|i-j| \leq 1$. Hence every chains of lines has arithmetic genus 0 and it is smoothable inside its ambient projective space. Fix any chain $X=C_{1} \cup \cdots \cup C_{d} \subset G(a, b) \subset \mathbf{P}^{N(a, b)}$ of $d$ lines. $X$ is smoothable inside $G(a, b)$, it is a smooth point of the Hilbert scheme $\operatorname{Hilb}(G(a, b))$ of $G(a, b)$ and $h^{1}\left(X, N_{X, G(a, b)}\right)=0\left([2]\right.$, Remark 2.5) For all $i$ the vector bundle $Q_{a, b} \mid C_{i}$ is a direct sum of a line bundle of degree 1 and $a-1$ line bundles of degree 0 . Using $d-1$ Mayer-Vietoris exact sequences we easily get $h^{0}\left(X, Q_{a, b} \mid X\right)=d+a$ and $h^{1}\left(X, Q_{a, b} \mid X\right)=0$. For all integers $a, b, x$ such that $1 \leq x \leq N(a, b)$ there is a chain $Y \subset G(a, b)$ of $x$ lines whose spans in $\mathbf{P}^{N(a, b)}$ has dimension $x$ ([2], Lemma 2.7).

Example 1. Fix integers $b>a>0$ and set $u:=\lceil N(a, b) / a\rceil$ and $\epsilon:=u a-$ $N(a, b)$. Hence $0 \leq \epsilon<a$. By Remark 2 there is a chain $T \subset G_{a, b}$ of $N(a, b)$ lines spanning $\mathbf{P}^{N(a, b)}$. Hence $\operatorname{dim}\left(V_{T, a, b}\right)=b$ and the determinantal map $\tau_{T, a, b}: \bigwedge^{a}\left(V_{T, a, b}\right) \rightarrow H^{0}\left(T, \mathcal{O}_{T}(1)\right)$ is bijective. By Remark $2 T$ is smoothable inside $G(a, b)$. Take a general such smoothing $C \subset G(a, b)$. Hence $C \cong \mathbf{P}^{1}$, 
$C$ spans $\mathbf{P}^{N(a, b)}$, and $\operatorname{deg}(C)=N(a, b)$. By semicontinuity $\operatorname{dim}\left(V_{C, a, b}\right)=b$ and the determinantal map $\tau_{C, a, b}: \bigwedge^{a}\left(V_{C, a, b}\right) \rightarrow H^{0}\left(C, \mathcal{O}_{C}(1)\right)$ is bijective. Identify $C$ with an abstract $\mathbf{P}^{1}$ and let $F$ be the rank $a$ vector bundle on $\mathbf{P}^{1}$ which is the direct sum of $\epsilon$ line bundles of degree $u-1$ and $a-\epsilon$ line bundles of degree $u$. Thus $F$ is the only rigid vector bundle on $\mathbf{P}^{1}$ such that $\operatorname{deg}(F)=\operatorname{deg}\left(Q_{a, b} \mid C\right)$ and $\operatorname{rank}(F)=\operatorname{rank}\left(Q_{a, b} \mid C\right)$. Since $Q_{a, b}$ is spanned and $C$ is smooth and rational, $h^{1}\left(C, Q_{a, b} \mid C\right)=0$. Thus $b=h^{0}\left(C, Q_{a, b}\right)=$ $h^{0}\left(\mathbf{P}^{1}, F\right) . Q_{a, b} \mid C$ is the flat limit of an integral family of vector bundles on $\mathbf{P}^{1}$ isomorphic to $F$. By semicontinuity we get the bijectivity of the determinantal $\operatorname{map} \bigwedge^{a}\left(H^{0}\left(\mathbf{P}^{1}, F\right)\right) \rightarrow H^{0}\left(\mathbf{P}^{1}, \mathcal{O}_{\mathbf{P}^{1}}(N(a, b))\right.$ is bijective.

Proof of Theorem 1. Set $\epsilon:=$ un $-N(n, b)$. Let $F$ be the vector bundle on $\mathbf{P}^{1}$ considered in Example 1. Fix a degree $k$ morphism $f: X \rightarrow \mathbf{P}^{1}$. Set $G:=f^{*}(F)$ and $V:=f^{*}\left(H^{0}\left(\mathbf{P}^{1}, F\right)\right) \subseteq H^{0}(X, G)$. Hence $\operatorname{rank}(G)=n$, $\operatorname{deg}(G)=u k n-k \epsilon, V$ spans $G$ and $\operatorname{dim}(V)=b$. By Example 1 the determinant $\operatorname{map} \tau: \bigwedge^{n}(V) \rightarrow H^{0}(G, \operatorname{det}(G))$ is injective. Since $d \geq g+u k n+1$, there is an effective and reduced degree $d-\operatorname{deg}(G)$ divisor $D$ such that $\operatorname{det}(G)(D) \cong L$. Let $E$ be the general vector bundle obtained from $F$ making $d-\operatorname{deg}(G)$ general positive elementary transformations, each of them supported by a different point of the support of $D$. The proof of [1], Cor. 2.4 and Prop. 2.7, gives the stability of $E$. Since $G$ is a subsheaf of $G$, we may see $V$ as a linear subspace of $H^{0}(X, E)$. Since $\tau_{G, V}$ is injective, $\tau_{E, V}$ is injective.

Proof of Theorem 2. The family $A$ is the family $\{\operatorname{det}(G)(D)\}$ in which $D$ varies in a non-empty open subset of the symmetric product of $d-u k n$ copies of $X$.

We work over an algebraically closed field $\mathbb{K}$. For the statement of Theorem 1 we require $\operatorname{char}(\mathbb{K})=0$.

\section{REFERENCES}

[1] E. Ballico, Brill-Noether theory for vector bundles on projective curves, Math. Proc. Philos. Soc. 124 (1998), no. 3, 483-499.

[2] E. Ballico, Curves in Grassmannians and Plücker embeddings: the injectivity range, Math. Nachr. 242 (2002), 38-45.

[3] G. P. Pirola and C. Rizzi, Infinitesimal invariant and vector bundles, arXiv:math.AG/0612813, Nagoya J. Math. (to appear).

[4] M. Teixidor i Bigas, Curves in Grassmannians, Proc. Amer. Math. Soc. 126 (1998), no. $6,1597-1603$.

Received: January 1, 2007 